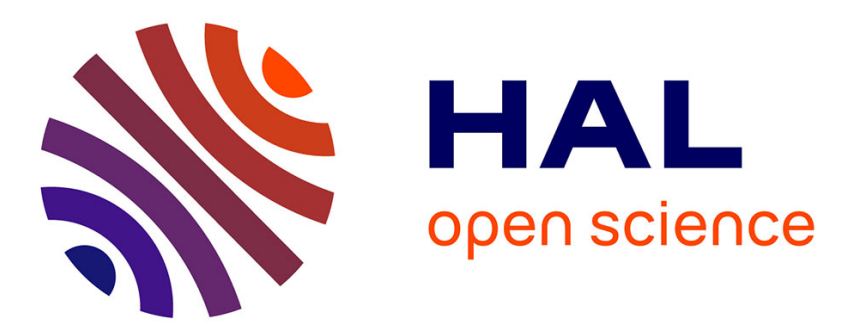

\title{
Le devenir-musée de la maison des Charmettes de Jean-Jacques Rousseau : la patrimonialisation d'une demeure à l'épreuve du temps
}

Hélène Cussac, Emmanuelle Lambert

\section{- To cite this version:}

Hélène Cussac, Emmanuelle Lambert. Le devenir-musée de la maison des Charmettes de Jean-Jacques Rousseau : la patrimonialisation d'une demeure à l'épreuve du temps. Culture et Musées, 2019, 34, pp.55-80. 10.4000/culturemusees.3632 . hal-03254130

\section{HAL Id: hal-03254130 \\ https://hal.science/hal-03254130}

Submitted on 8 Jun 2021

HAL is a multi-disciplinary open access archive for the deposit and dissemination of scientific research documents, whether they are published or not. The documents may come from teaching and research institutions in France or abroad, or from public or private research centers.
L'archive ouverte pluridisciplinaire HAL, est destinée au dépôt et à la diffusion de documents scientifiques de niveau recherche, publiés ou non, émanant des établissements d'enseignement et de recherche français ou étrangers, des laboratoires publics ou privés. 


\section{OpenEdition}

\section{Culture \& Musées}

Muséologie et recherches sur la culture

$34 \mid 2019$

Maisons-musées. La patrimonialisation des demeures des illustres

Dossier

\section{Le devenir-musée de la maison des Charmettes de Jean-Jacques Rousseau : la patrimonialisation d'une demeure à l'épreuve du temps}

The museum-becoming of J.-J. Rousseau's home of Charmettes. The patrimonialization of a house in the long term

La trasformación en museo de la Maison des Charmettes de Jean-Jacques Rousseau

HéLÈne CussaC ET EMmanuelle LAMBERT

p. $55-80$

https://doi.org/10.4000/culturemusees.3632

\section{Résumés}

Français English Español

La maison des Charmettes, à Chambéry, est l'une des demeures de Jean-Jacques Rousseau qui devint dès le XVIIIe siècle un lieu de pèlerinage reconnu. Classée monument historique au début du XXe siècle, récemment labellisée " Maison des illustres ", elle est l'objet de dénominations qui s'accompagnent d'une volonté de valoriser, d'interpréter, voire de re-créer le passé rousseauiste. Entre patrimoines matériels et immatériels tenant à l'écrivain, cet article retrace le processus de patrimonialisation du temps même du philosophe à nos jours, des pèlerinages aux témoignages sur les livres d'or et aux choix muséographiques et de médiation, lesquels font preuve d'une construction patrimoniale par les visiteurs autant que par l’institution.

The Charmettes's House in Chambery is one of the dwellings of Jean-Jacques Rousseau, which became a place of renown pilgrimage starting in the $18^{\text {th }}$ century. Classified as a Historic Monument at the beginning of the $2^{\text {th }}$ century, it was recently named the "House of Illustrious." It is the object of denominations that come from a desire to value, interpret and even re-create the past of Rousseau. Between material and immaterial heritage of the writer, this publication traces the process of patrimonialism from the philosopher's time to today, from the pilgrimages, testimonials of the gold books to the choices of museographic and mediation, wich show a patrimonial construction made by the visitors as well as by the institution. 
La Maison des Charmettes, en Chambéry, es una de las residencias de Jean-Jacques Rousseau, que se convirtió en un reconocido lugar de peregrinación en el siglo XVIII. Clasificado como Monumento Histórico a principios del siglo XX, recientemente etiquetado como «Casa de Ilustres», es objeto de denominaciones que van acompañadas de un deseo de valorizar, interpretar e incluso recrear el pasado Rousseoista. Entre el patrimonio material e inmaterial ligados al escritor, este artículo recorre el proceso de patrimonialización, desde la época del filósofo hasta nuestros días, desde las peregrinaciones hasta los testimonios sobre los Libros de Oro, pasando por las decisiones museográficas y de mediación, que demuestran la construcción de un patrimonio tanto por parte de los visitantes como de la institución.

\section{Entrées d'index}

Mots-clés : nationalisation, patrimonialisation, médiation, maison-musée d'écrivain, Rousseau (Jean-Jacques)

Keywords: nationalisation, patrimionalism, mediation, writer's house-museum, Rousseau (Jean-Jacques)

Palabras clave: nacionalización, patrimonialización, mediación, casa-museo de escritor, Rousseau (Jean-Jacques)

\section{Notes de la rédaction}

Manuscrit reçu le 24 mars 2019

Version révisée reçue le 25 octobre 2019

Article accepté pour publication le 30 octobre 2019

\section{Texte intégral}

1 Parmi les nombreuses demeures où résida Jean-Jacques Rousseau, la maison des Charmettes, à Chambéry attire particulièrement l'attention, d'une part parce qu'elle est à l'origine de son « magasin d'idées » (Rousseau, 1959 : 237), d'autre part parce qu'elle est devenue non seulement un lieu de tourisme culturel, mais surtout, en raison du culte précoce voué au philosophe, le lieu de pèlerinage qu'elle est encore aujourd'hui. Parmi les habitats rousseauistes, son évolution est, d'un point de vue mémoriel, l'exemple même de l'importance du lien entre la maison d'un écrivain, son œuvre, et ceux qui souhaitent en conserver le souvenir ${ }^{1}$; et d'un point de vue institutionnel, l'exemple d'un devenir-patrimoine. Patrimoine immatériel, en ce que la demeure est liée à la figure d'un écrivain qui n'est plus mais que l'on continue à vénérer ; patrimoine matériel, du fait de l'existence de la maison, de son ambiance et de son environnement naturel participant à la construction mémorielle.

2 La maison des Charmettes fut classée monument historique le 10 mars 1905 ; le vallon qui l'abrite, en 1933. Puis elle reçut le label « Maison des illustres » en 2012, année du tricentenaire de la naissance du philosophe. Ces étapes montrent que les conservateurs et les instances publiques eurent conscience très tôt du fait que cette demeure était un bien patrimonial à sauvegarder. Appelée aussi bien Maison des Charmettes que Musée des Charmettes - y aurait-il là quelque ambiguïté à interroger ? -, quitterait-elle alors son statut de maison d'écrivain ${ }^{2}$ en s'affirmant davantage comme un musée grâce au soutien des instances culturelles de la ville, comme en témoigna l'inauguration de l'Espace Jean-Jacques Rousseau - salle d'interprétation des Charmettes les 15 et 16 septembre 2018 lors des Journées du patrimoine ? Le musée que la demeure est appelée à devenir, avec les médiations qu’il implique, permettra-t-il davantage encore le travail mémoriel et l'appropriation de l'œuvre par le public?

3 Cette maison où résida Rousseau entre 1736 et 1742 , et où il connut avec Mme de Warens son premier amour - élément biographique non neutre dans l'action de mémorisation -, participe de tout un processus de patrimonialisation qui s'accompagne d'une volonté certaine d'interpréter, voire de recréer le passé à la lumière des différents moments de cette mise en perspective patrimoniale. C'est ce processus qui est ici examiné sous un angle tout d'abord historique, du mouvement qui, du culte envers Rousseau, a conduit jusqu'à nos jours de plus en plus de visiteurs à accomplir un véritable pèlerinage sur ses pas. Nous avons ensuite observé la part prise par les 
visiteurs dans le processus de patrimonialisation, tant du vivant de Rousseau, qu'après son décès à partir des témoignages dans les livres d'or. Enfin, pour mieux cerner la patrimonialisation en acte, examiner la visite qui est de nos jours offerte aux publics, à travers des actions de valorisation et de médiation, permet d'illustrer ce processus encore à l'œuvre.

\section{Culte, pèlerinage et commémorations : le présage d'un rayonnement patrimonial}

Si d'aucuns connaissent les éléments historiques que nous rappelons ci-dessous, ils permettent de mieux entendre le processus de patrimonialisation qui fit passer un écrivain du XVIIIe siècle à la figure d'illustre et à l'élaboration de ses modestes lieux de vie en sites patrimonialisés.

\section{Un culte précoce}

Adulé ou conspué dès son vivant, Rousseau ne laissait personne indifférent. Faut-il rappeler aussi que ses écrits, particulièrement ceux qui prêtèrent à polémique, voire à censure, firent grand bruit dans l'univers savant et la sphère publique ? De même que sa marginalité radicale du fait de sa manière de vivre, dans une solitude revendiquée en un siècle qui prônait la sociabilité, souleva l'ire de ses pairs philosophes ? La relation à l'écrivain fut en effet sans cesse manichéenne, mais sa pensée singulière, d'une portée philosophique et politique majeure, participa très tôt à une adulation sans bornes : « $\mathrm{A}$ la veille de la Révolution, le culte de Jean-Jacques existe depuis une décennie » (Barny, $1986: 98)$.

$6 \quad$ Mais ce ne fut pas seulement par ses écrits que Rousseau, exposé malgré lui à la publicité, connut de son temps le succès. Peu de temps après avoir été couronné par l'Académie pour son Discours sur les arts et les sciences (1750), il assiste aux deux représentations de son opéra, Le Devin de village, données à Fontainebleau devant le roi et la cour, qui furent une réussite. Rousseau n'était donc pas seulement un être aux multiples talents de philosophe, romancier, compositeur et auteur d'essai pédagogique, il était déjà un grand homme de la nation : "Chacun de ses livres [était] un événement. Aucun autre écrivain du siècle n'aura trouvé aussi indiscutablement un public [...] JeanJacques fut donc l'auteur le plus à la mode » (Bonnet, $1998: 199$ ).

$7 \quad$ Au-delà du culte se construit donc progressivement un véritable mythe qui oeuvre pour la postérité. Ses écrits, notamment Du contrat social, l'Émile et les Lettres écrites de la montagne, sont perçus comme sulfureux et entretiennent la légende : " [Rousseau] est comparé à Socrate par plusieurs de ses correspondants » (ibid. : 207). Son entrée dans la «fabrique des illustres » est dès lors effective en ce que le philosophe est devenu « une personne célèbre considérée comme un événement [déjà] façonné par des médiations " (Saurier, 2013). Ces médiations, terme certes anachronique pour l'époque, correspondent à ses éditeurs, à la presse, aux correspondances, aux lecteurs, mais aussi aux scandales et aux sanctions que suscite son œuvre.

8 Ce patrimoine en devenir fut consolidé par la réalisation de bustes, de portraits ${ }^{3}$, de gravures dont le succès fut considérable. De nombreuses publications au sujet de l'œuvre, la collection de ses manuscrits, sa panthéonisation grandiose et les diverses commémorations qui s'ensuivirent contribuèrent à élaborer « les constantes notables [qui] fondent [aujourd'hui] l'unité des musées littéraires » à partir des " quatre unités sur lesquelles travaillent toutes les Maisons d'écrivain » : « un auteur - une œuvre une époque - un lieu » (Dekiss, 2009: 784). 


\section{La destinée d'un personnage}

$9 \quad$ Durant les seize années qui séparent le décès du philosophe de son entrée au Panthéon, sa célébrité ne fait que croître, entraînant de multiples projets dont celui, en 1885, de lui élever une statue place du Panthéon, qui verra le jour l'année du centenaire de la Révolution. "À partir de cette date et jusqu'au bicentenaire, les partisans de Rousseau vont agir et multiplier les hommages à cet auteur en France. Chaque ville qui a connu le séjour du philosophe fournit l'occasion d'inaugurer une statue » (L'Aminot, 1992 : 11). La célébrité ne suffit pas, il s'agit de le rendre visible, au sens de Nathalie Heinich (2012). Les monuments à Jean-Jacques Rousseau s'élèvent tour à tour 4 et donnent lieu à des inaugurations très politisées. Sans grand bruit toutefois, « le premier monument où figura Rousseau fut réalisé dans un contexte éloigné de toute référence funéraire, à Genève, alors même que l'écrivain vivait encore » (Scherf, $2012: 158$ ). Tout autant qu'en France, le citoyen de Genève a ses admirateurs suisses qui témoignent ainsi de leur volonté de l'inscrire dans la mémoire collective nationale.

De multiples publications accompagnent cette période d'hommages à Rousseau. La Révolution ayant fait du philosophe le père de la Convention, en juillet 1790, l'Assemblée constituante décrète que son buste sera placé dans la salle des séances, et en 1791 paraît, sous la plume de Louis-Sébastien Mercier, De J.-J.Rousseau considéré comme l'un des premiers auteurs de la Révolution. Un siècle plus tard, en 1887, avec l'ouvrage de Castellant 5 intitulé $J .-J$. Rousseau : hommage national, le philosophe est bel et bien devenu une figure de la nation. Les biographes ne pouvaient pas rester insensibles aux séjours de Rousseau aux Charmettes ; aussi relève-t-on une contribution au titre de Madame de Warens et J.-J. Rousseau (1890). Tanguy L'Aminot indique que 15000 ouvrages d'art et d'essais lui furent consacrés entre 1900 et 1992. La dimension historique, quantitative et qualitative des opérations, tant du côté de la sphère publique et politique que savante, rend particulièrement sensible le passage d'un écrivain et la totalité de ce qui le concerne au statut de bien national et patrimonial. Le cas de Rousseau est de ce point vue exemplaire : en 1893, il est l'un des premiers auteurs à paraittre dans la collection « Les grands écrivains français ». L'œuvre rousseauiste est bien un « immense capital de mémoire collective » (Nora, $2011: 374$ ), et l'école en sera un passeur primordial.

Ce capital, il s'agissait toutefois de le rassembler pour qu'il entre justement au patrimoine de la nation, et en 1794, en raison à la fois « de l'intérêt grandissant pour les manuscrits littéraires d'une part, et pour l'extrême popularité du philosophe de l'autre », on relève " une effervescence particulière autour des papiers de Rousseau » (Sacquin, 2012 : 57). On ne peut donc être surpris d'apprendre que l'expression " patrimoine national » au sujet de manuscrits apparait à ce moment-là (ibid.). " Il est un autre monument à élever à la gloire de J.-J. Rousseau », déclare d'ailleurs Boissy d'Anglas, " c'est de déposer ses manuscrits dans la Bibliothèque nationale » (Guillaume, 1891-1958, t. VI : 938). Ces manuscrits, traces concrètes du génie, sont des objets signifiants que la nation se doit de préserver. Leur sauvegarde en 1794 signe la volonté mémorielle d'enraciner l'écrivain dans le patrimoine littéraire, culturel et sociopolitique (Ferrand, 2012), pendant que les restes de Rousseau sont « déclarés propriété nationale » (Sacquin, $2012: 61$ ). Mais ce travail d'une mémoire émancipatrice, dont tout l'enjeu est la démocratie, occasionna des polémiques telles que la postérité de l'homme, de l'œuvre et des lieux pouvait s'en trouver fragilisée : "L'intégration au patrimoine d'un magicien du langage doublé d'un prophète errant ne se fait pas sans débats, combats, mutations et paradoxes qui révèlent la complexité de l'enjeu Rousseau », comme l'expriment Jean-Marie Goulemot et Éric Walter (1984 : 410).

Rousseau, très tôt figure nationale, est aussi devenu une figure patrimoniale malgré les opposants à cette reconnaissance. Le processus s'effectue au fil d'événements du présent qui donnent lieu à des conflits et des réinterprétations du passé ; ainsi se crée et se recrée dans la sphère publique celui qu'on a érigé en personnage : "Les personnes célébrées sont entourées d'une aura qui puise dans un substrat culturel appartenant à la mémoire collective ", remarque à juste titre Delphine Saurier (2013). Comme l'ont montré les manifestations pour le tricentenaire de sa naissance (2012), Rousseau reste 
donc " étonnamment vivant », comme il l'était déjà cinquante ans auparavant (Baudoin, 1962 : 19). Mais de bonne heure aussi, les lieux rousseauistes ont connu une affluence de visiteurs, qui doit être interrogée dès lors que l'on s'intéresse à la fortune d'une œuvre et à son public, celui-ci participant à la construction patrimoniale.

\section{Les visiteurs dans le processus de patrimonialisation}

Une des " nouveauté[s] du siècle des Lumières consiste [...] à transposer au monde des vivants, sans jamais les abandonner, des pratiques rituelles jusqu'ici réservées à l'hommage posthume » (Guichard, 2009: 12). Rousseau est si célèbre qu'il suscite soit un intérêt intellectuel, soit une curiosité plus ou moins malsaine, mais toujours est-il que beaucoup désirent le voir.

\section{Les visites du vivant de Rousseau, ou la publicité d'une image}

Très tôt, le terme de pèlerinage, qu'il aurait lui-même lancé dans les Confessions au sujet de ces visites (Rousseau, 1959 : 527), signifie bien la dimension sacrée donnée à l'acte de visite. Ses visiteurs sont toujours décrits comme des pèlerins, et les lecteurs font déjà preuve dans leurs lettres à " l'ami Jean-Jacques » d'un réel « transfert affectif » (Lilti, $2014: 165$ ).

15 Des passages de l'œuvre, la correspondance ainsi que des témoignages écrits donnent des informations précieuses sur les visiteurs du temps de Rousseau. Certes, les spécialistes ont déjà bien étudié la question ${ }^{6}$, qui soulève néanmoins de l'intérêt du point de vue du processus de patrimonialisation. Le récit de la rencontre de Rousseau en son temps est en soi un événement : " On est frappé par le succès considérable du genre littéraire auquel donna lieu la pratique des "Relations de la visite à J.J. Rousseau" » (Berchtold \& Porret, 1999 : 7). Ce témoignage de l'expérience de visite dans un contexte historique précis joue un rôle à la fois de créateur et de passeur :

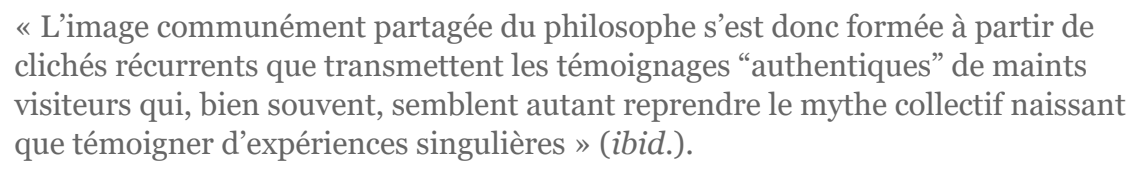

Le statut de ces traces de mémoire individuelle in praesentia est bien testimonial. Le témoignage est ainsi lui-même un médiateur entre la personne illustre que l'on a façonnée en événement, quitte à la défigurer (Lilti, 2014 : 206), et les admirateurs postérieurs. De ce fait, il participe à part entière du processus du devenir-patrimonial de et autour de Jean-Jacques.

Le penseur atypique attire, et les traces de ces visites sont inestimables. C'est le cas, par exemple, de Jacques Wegelin et Jean Gaspard Schulthess, deux admirateurs qui, en octobre 1763 , après plusieurs entretiens, rédigent un document dont plusieurs versions circuleront de manière confidentielle ${ }^{7}$ et contribueront au mythe. Des aristocrates (le prince de Ligne, la princesse Isabelle Czartoyska), des savants (le médecin Samuel Tissot), des écrivains (Goldoni, Bernardin de Saint-Pierre), mais aussi des gens du peuple font le déplacement. Rousseau lui-même en témoigne :

« J'avois à Motier presque autant de visites que j'en avois eu à l'Hermitage et à Montmorenci, mais elles étoient la pluspart d'une espéce fort différente. Ceux qui m'étoient venu voir jusqu'alors étoient des gens [...] ayant avec moi des rapports de talens, de gouts, de maximes [...]. À Motier [...] [c]étoient des officiers et d'autres gens qui n'avoient aucun gout pour la littérature, qui même pour la pluspart n'avoient jamais lû mes ecrits [...]» (Rousseau, 1959 : 611). 
"C'est lors de son retour à Paris, en 1770, tellement annoncé et commenté par la presse, qu'il fut le plus assailli »(Bonnet, 1998 : 211). Quelques témoignages font comprendre combien la fascination du public envers Rousseau reflète la construction d’un personnage devenu mythique : « [...] sa présence [au café de la Régence] a attiré une foule prodigieuse [...]. On demandait à la moitié de cette populace ce qu'elle faisait là ; elle répondait que c'était "pour voir Jean-Jacques"8. » La destinée posthume était prévisible : à la mort du philosophe, le 2 juillet 1778 , c'est une quasi-divinité que l'on honore et à laquelle on rendra visite sur tous les lieux qu'elle a occupés.

\section{L'ampleur des visites et l'émergence d'une médiation}

Le poète Roucher est l'un des premiers, en août 1778, à exprimer sa ferveur par un éloge funèbre qui se répand dans Paris ${ }^{9}$. L'un des lieux premiers du culte, tout au long du moment révolutionnaire, est l'île des Peupliers dans le parc d'Ermenonville, où se trouve la tombe de Rousseau. Il s'agit là d'un parc à l'anglaise que le marquis de Girardin, médiateur du patrimoine rousseauiste avant l'heure, modèle selon un parcours « en fonction des exigences mêmes du culte [...]. Ainsi, on n'accède au maîtreautel qu'après de nombreuses stations : un pèlerinage bien conçu peut se prolonger plusieurs jours » (Barny, 1986 : 98). Les visites à Ermenonville deviennent un rituel, au point que Pierre Le Tourneur publie en 1788 un Voyage à Ermenonville, véritable guide du pèlerin, et que paraît en 1799 un Voyage à l'Isle des Peupliers. "Dès le tournant du siècle, la visite a ses littérateurs et ses brochures touristiques » (Guichard, 2009 : 17). Georges-Marie Raymond, devenu propriétaire de la maison des Charmettes en $1810^{10}$, publie une Notice sur les Charmettes, vallon des environs de Chambéry ; à l'usage des voyageurs qui visitent la retraite de J. J. Rousseau : son objectif est la composition d'un guide touristique. Même si des décennies séparent Rousseau de l'écriture de ses grands textes, la maison des Charmettes et son vallon sont les lieux les plus marquants de sa vie et de l'œuvre à venir.

$\mathrm{Du}$ pèlerinage philosophique et patriotique émerge ainsi le pèlerinage littéraire, lequel « entretient la fabrication industrielle de portraits, objets et bustes du philosophe que chacun place dans son univers domestique » (Berchtold \& Porret, 1999: 17). Se rendre chez Rousseau, le rencontrer par l'imagination et l'émotion est donc une démarche indispensable. Le citoyen de Genève est entré définitivement dans le patrimoine ; telle devait être sa destinée, comme le souligne Germaine de Staël, dont le premier écrit est consacré à Rousseau : " La gloire des grands hommes est le patrimoine d'un pays libre, après leur mort le peuple entier en hérite » (1998: 326). C'est l'homme illustre que l'on vient honorer lors de la visite à Ermenonville ; c'est à la fois du philosophe et de l'homme tout simplement que Français, Suisses, mais encore Européens et internationaux, célèbres ou anonymes, viennent se souvenir aux Charmettes. Beaucoup accomplissent le pèlerinage rêvé et marchent sur « les chemins de Jean-Jacques », ainsi que s'est intitulée l'exposition en deux volets de 2011 et 2012 à la Maison des Charmettes. Beaucoup remplissent aussi les livres d'or, dont le premier fut mis à disposition des visiteurs par Georges-Marie Raymond en 1810. On ne saurait négliger cette forme de document auquel ce premier conservateur de la maison attache beaucoup d'importance :

\footnotetext{
«En mon absence, les fermiers sont chargés de présenter le registre aux personnes dont ils satisfont la curiosité en leur ouvrant la maison. Je regrette beaucoup que cette idée assez naturelle n'ait pas été mise à exécution par les propriétaires qui m'ont précédé » (1811: 289-290).
}

Quelles que soient en effet les diverses émotions qui s’y expriment : admiration, fétichisme, idolâtrie, rejet ou haine, la trentaine de livres d'or des Charmettes sont riches d'enseignement sur la réception et la perception de Rousseau en des contextes historiques et socio-politiques singuliers. De ce fait, ils sont le témoin de mémoires individuelles liées à la mémoire collective scolaire, sociale et politique, et ainsi passeurs du patrimoine rousseauiste, tant matériel qu'immatériel, au sens où « la continuité 
entre le passé et le présent est assurée par l'intermédiaire des individus eux-mêmes » (Davallon, 2015 : 27).

\section{Le livre d'or des visiteurs ou un document co- constructeur de la patrimonialité}

La maison des Charmettes est par conséquent l'expression d'une identité littéraire et d'une forme de patrimonialité, entendue comme « le rapport d'attachement des pratiquants ordinaires d'une ville avec ce qu'ils considèrent comme leur patrimoine » (Watremez, 2008 : 12). Cette notion de patrimonialité, qui n'est autre qu'une relation subjective aux lieux de vie et à l'espace urbain - selon Watremez, mais ici plus bucolique -, peut se traduire par la place symbolique accordée aux publics, notamment par les traces et écrits de visiteurs sur les livres d'or. L'étude diachronique des écrits de visiteurs issus de douze volumes (de 1820 à 2017) sur les 29 que constitue la collection des livres d'or des Charmettes a en effet permis d'observer la diversité des témoignages selon les époques ${ }^{11}$. À travers ces traces, l'idée de transmission patrimoniale prend corps. Le patrimoine rousseauiste est mis à l'épreuve du temps comme le souligne ce témoignage datant de 1927 sur l'un des livres d'or :

\footnotetext{
« Est-ce là le site, seulement qui nous enchante et nous entraîne vers la rêverie ? $\mathrm{Ou}$, est-ce un restant de vie qui surgit tout à coup devant nous ? Est-ce tout un passé qui surgit devant moi ? Je ne puis m'expliquer la cause de mon émoi. »
}

La relation au présent, au passé et à la mémoire se trouve bouleversée, car ce qui caractérise la maison d'un écrivain, a contrario d'un musée, est qu'elle a été habitée et que la sauvegarde de l'authenticité donne l'illusion d'entrer dans l'intimité de l'auteur. Dans le cas de la maison des Charmettes, ce pouvoir magique des lieux agit, et c'est bien de transmission patrimoniale qu'il est question, d'un patrimoine cette fois-ci de valeurs, morales et politiques, à lire cet autre témoignage du 2 juin 1860 :

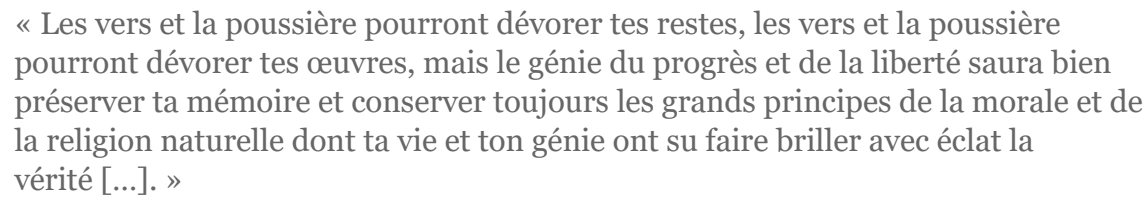

La conscience du temps qui passe chez ce visiteur n'empêche pas l'abolition de toute distance temporelle avec Rousseau, comme le signifie le tutoiement, très souvent utilisé dans ces registres. Ce qui sert aussi la construction, la « fabrique du patrimoine » (Heinich, 2009), ce sont l'émotion (Heinich, 2012) et l'attachement (Hennion, 2004 ; Tornatore, 2010) que mobilise le visiteur et qui transparaissent dans son témoignage. Quelle que soit la date de la visite, elle correspond pour ce dernier à un parcours de l'intime, à une impression de véritable rencontre, ainsi que l'exprime un visiteur sur une page d'août 1927. S’y lit non seulement de l'affectivité, mais se révèle à travers son sentiment d'effacement du temps celui de contribution au patrimoine :

\footnotetext{
« M'arrêtant devant chaque objet, chacun me dit un je ne sais quoi, que je ne puis définir. Le temps a effacé certaines traces, mais il en est une qui, pour moi, n'est point effacée : c'est un restant de vie qui, malgré les années, ne peut point s'effacer. En étudiant bien ce qui se passe en moi, je ne puis appeler cela du souvenir. Je ne pourrais que dire, que ce qui fut un temps, est aujourd'hui encore, car un restant de vie subsiste en ce nid. »
}

La continuité entre le temps de Rousseau et le temps du visiteur est effective. Travaillent à ce fil rouge des mémoires à la fois le souvenir de l'œuvre et l'existence de la demeure. C'est la raison pour laquelle les livres d'or des Charmettes prennent la dimension de bien commun et patrimonial. Nombre de ceux qui y ont couché leur plume semblent bien l'avoir fait dans le sens de la transmission : " le blé que tu as semé lève encore - écrit ce visiteur du 17 septembre 1941 -, comme l'a dit Bazin tu seras le guide et la lumière de beaucoup de générations encore ». Les traces sur ces registres 
vont bien au-delà d'un hommage traditionnel, plus distancié. Certes, l'admiration et la reconnaissance s'y lisent souvent, mais le vœu de conserver la mémoire du philosophe n'est pas un souhait formulé à la légère : " Je n'ai pas voulu passer à Chambéry sans venir visiter la demeure de l'illustre Jean J. Rousseau, pour en garder le souvenir à perpétuité » (un visiteur en 1860). Il arrive même que le visiteur fasse le lien avec l'avenir dans le sens où Rousseau est, dans les périodes politiquement troubles, encore un garant de liberté démocratique : "C'est ici que dans la beauté simple de la nature savoyarde se sont épanouis et révélés le génie et la sensibilité d'un de nos plus grands auteurs, Jean-Jacques Rousseau, dont la pensée a changé le monde et dont la lecture peut ainsi nous protéger des menaces qui nous entourent », écrit François Hollande sur le livre d'or en 2016.

Ces registres revêtent par conséquent la fonction de co-construction d'un patrimoine non figé, sans cesse réactualisé :

\footnotetext{
«Les livres d'or des Charmettes sont ainsi des documents qui représentent une double valeur : testimoniale et patrimoniale, et qui opèrent une double patrimonialisation, dans le sens d'une double mise en mémoire : celle matérielle du fonds des livres d'or, et celle plus immatérielle, liée à la figure de Rousseau et aux visiteurs » (Cussac \& Lambert, 2020a).
}

Que vive encore cette maison est apparu en ce sens indispensable à tous ses conservateurs, du tournant des Lumières jusqu'à nos jours, d'où un travail de réactualisation permanente de ce patrimoine par autant de gestes muséographiques que de propositions de médiation.

\section{Valorisations contemporaines au service du devenir-patrimoine}

La patrimonialisation est un processus (Rautenberg \& Tardy, 2013), une construction faite de gestes et de réinterprétations (Davallon, 2006), tout autant que la médiation qui consiste précisément à réactualiser ou faire revivre un objet, un patrimoine, à réinvestir le passé par et dans le présent. S'il existe des constructions réciproques entre des figures de personnes célèbres et des lieux de vie historiques devenus patrimoniaux, comme l'a démontré Delphine Saurier (2003, 2007), dans quelle mesure les travaux, les changements muséographiques contemporains et les médiations sensibles mettent-ils en valeur ou restructurent-ils ce patrimoine?

\section{Restructuration d'une maison-musée à l'ambiance authentique?}

Le changement récent du statut des Charmettes, labellisée « Maison des illustres » en 2012, comme nous l'avons évoqué, s'accompagne, selon les supports de communication, de dénominations diverses : elle est en effet dite tour à tour Maison des Charmettes, Musée des Charmettes ou Maison de Jean-Jacques Rousseau ${ }^{12}$. Cette instabilité des noms est le signe d'un partage délicat entre deux sphères : celle de l'intime, la maison étant le cœur de l'intimité, du vécu, alors que le musée réfère davantage à la sphère publique et à une légitimation, voire une sacralisation. Le point de convergence consiste notamment en ce qu'une maison d'écrivain et un musée usent de techniques muséographiques et transforment la relation aux publics : « La Maison d'un écrivain déplace la relation écrivain-lecteur vers une relation élargie de l'écrivain au public » (Dekiss, 2009 : 783). La nouvelle dénomination peut traduire aussi un changement de statut symbolique : de maison sans doute modeste ancrée dans le passé, elle deviendrait plus noble en s'affirmant musée ; ou a contrario perdrait-elle son âme en se muséifiant? La présentation des Charmettes, sur le site Internet de la ville de Chambéry, comme " musée d'ambiance et de souvenir littéraires » semble affirmer le 
contraire. La demeure a en effet connu des rénovations et transformations sur le plan architectural et muséographique qui ont accompagné ce changement.

Les aménagements architecturaux, pour revenir à la configuration originelle des lieux, les petites pièces ouvertes sur l'extérieur et un certain dénuement décoratif préfigurent sans doute une première impression ou interprétation sur l'œuvre. Ils témoignent aussi d'une humilité dans la conservation et la fonction même du lieu : c'est une maison avant d'être un musée. La quasi-absence de panneaux, feuilles de salle ou cartels dans les pièces semble ainsi les préserver, comme si le temps était suspendu. Or, si la préservation d'ambiances du passé renforce encore davantage la sanctuarisation, voire la muséification des lieux, il est intéressant de noter que les décors intérieurs et les chambres ont été reconstitués. En effet, si l'atmosphère du bonheur selon Rousseau s'incarne dans cette demeure, celle-ci a perdu son état originel ; les reconstitutions font illusion en cherchant à traduire une image ressemblante ou fidèle à l'esprit de l'époque. Mais l'essentiel est ailleurs, dans l'ambiance ainsi recréée ; et l'ambiguïté entre vrai et faux semble secondaire. De plus, les espaces d'habitation et la récente salle d'interprétation sont séparés, notamment dans leurs rôles : la demeure et son atmosphère surannée privilégient les émotions, tandis que l'espace plus proprement muséographique, moderne et dépouillé, développe le "magasin d'idées » pour une meilleure compréhension. Enfin, pour clarifier cette confusion des dénominations, mentionnons que si cette maison d'écrivain est bien une demeure patrimonialisée, en l'absence de collection elle n'en constitue pas pour autant un musée, tel qu'on l'entend aujourd'hui.

Néanmoins, toute maison d'écrivain est un lieu unique, où " chaque objet donne à voir, à ressentir ou à interroger l'environnement de l'homme célèbre. Chaque indice pris individuellement retisse des fils invisibles qui relient l'œuvre à l'espace de vie » (Scipion, 2009). La recherche de l'authenticité s'exprime ainsi à travers des choix muséographiques de conservation et de restitution qui préservent et garantissent cette relation indicielle au passé. La mise en exposition d'objets, de documents et de mobilier ancien, ainsi que la scénographie dépouillée construisent une première forme de médiation. Si les restaurations de la maison des Charmettes proposent des espaces aussi authentiques que possible, c'est notamment pour offrir une découverte sensorielle fondée sur l'émotion (Cussac \& Lambert, 2020b). Historiens, sociologues et muséologues ont en effet montré que la relation au patrimoine s'ancre dans une relation émotionnelle ${ }^{13}$. Pour David Sander et Carole Varone (2011), le lieu d'exposition est au service des fonctions émotionnelles autant que l'émotion aide le lieu à remplir ses fonctions muséales, ce qui s'affirme encore davantage pour les maisons d'écrivains où " [i]l apparaît qu'au-delà du ressort de la connaissance littéraire, c'est le ressort de l'émotion, de l'imaginaire qui conduit le visiteur d'une maison d'écrivain à vouloir découvrir et s'imprégner des lieux » (Bonniot-Mirloup \& Blasquiet, 2016). À l'image d'autres musées, tels ceux d'histoire, l'émotion est un levier de médiation, voire de patrimonialisation : « l'émotion exprimée par le visiteur est située, elle lui permet de se projeter dans l'événement historique, de s'identifier à l'époque, de retrouver des souvenirs » (Ancel, $2015: 36$ ). Le désir de curiosité et le besoin de mémoire des publics s'expriment, comme on l'a observé dans les témoignages inscrits dans les livres d'or des Charmettes, par des émotions de toutes sortes. La singularité des maisons-musées d'écrivains se manifeste alors par deux temporalités : la façon dont le lieu correspond à la fabrique de l'œuvre en son temps, et celle dont il continue de témoigner de la fabrique du patrimoine dans le présent. Dans ce processus de construction puis de transmission, quel rôle peut jouer la médiation sensorielle pour continuer de faire vivre un peu de l'esprit du lieu et de l'œuvre?

\section{Les médiations contemporaines dans le processus de patrimonialisation}

Dès les années 1970, la Ville de Chambéry se dote d'un service éducatif - devenu depuis service des publics - qui développe une politique centrée sur l'éducation 
artistique et culturelle pour les trois musées dont elle a la gestion. Ainsi, comme l'affirme Mireille Védrine dans le projet scientifique et culturel des Charmettes :
« [Cette demeure est un] lieu de mémoire, d'expérimentation, d'apprentissage et d'éducation original, pour les jeunes et pour les adultes, qui défend des valeurs universelles, un lieu où l'on peut apprendre, en dehors des institutions et des médias, à travers Rousseau et ceux qui se sont inspiré de lui, à mieux comprendre la nature, le bonheur, la société et l'Homme, une "école buissonnière", en grande partie autodidacte, "une éducation dont le cœur se mêle", une leçon philosophique, qui s'appuie sur les textes, mais aussi sur l'expérience sensible d'un lieu, l'itinéraire d'un homme, et sur l'émotion14. »

Les médiations actuelles mises en œuvre aux Charmettes donnent du sens au programme muséographique de ce lieu de mémoire en suscitant, d'une part, des réminiscences émotionnelles et stimulant, d'autre part, des expériences sensorielles : " La notion de médiation permet ainsi de comprendre autrement le processus de patrimonialisation [...]. L'objet culturel apparaît alors continuellement travaillé dans son sens (et dans sa forme dans une moindre mesure) par une médiation » (Saurier, 2007).

Certaines modalités de médiation correspondent à une approche classique dans la mesure où.
« [...] la médiation [y] requalifie le patrimoine comme un objet à contempler ou à comprendre, au sein d'un monde à explorer, à découvrir : elle propose un point de vue sur le patrimoine à travers la configuration d'un dispositif de représentation et de communication qui oriente les visiteurs vers une mise en signification - des lieux, des patrimoines » (Flon, $2014: 14)$.

Mentionnons ainsi des médiations écrites lors des commémorations de 2012 : par exemple le livret Rousseau 2012 Chambéry, sous-titré Chambéry et les Charmettes célèbrent l'année Rousseau, qui développe une programmation annuelle éclectique et tous publics ; ou les journaux Les Chemins de Jean-Jacques (sous-titrés Petit journal de l'exposition) qui accompagnent et explicitent les expositions. Mentionnons encore la production éditoriale de catalogues savants richement illustrés ${ }^{15}$ qui œuvrent à la connaissance de ce patrimoine et ainsi à sa mémoire.

Des propositions assez habituelles se déclinent régulièrement, comme en témoignent les plaquettes-programmes de 2017 et $2018^{16}$ : la visite accompagnée et la visite audioguidée, le parcours historique et ludique avec tablette numérique «Sur les pas de Jean-Jacques Rousseau ", des conférences ou le goûter-philo, des livrets-jeux et des ateliers, etc. La salle d'interprétation complète ces médiations centrées sur une approche éducative : l'intention de transmission de savoirs et la mission d'élargissement des publics y sont à l'œuvre. La diversité des situations de médiation prolonge la construction patrimoniale de cette demeure. Il s'agirait alors davantage d'une co-construction symbolique, car celle-ci « suppose des actions qui, de façon conjointe et/ou additionnée, font advenir ou évoluer les représentations du patrimoine, impliquant autant les interactions directes que les formes indirectes » (Saurier, 2003).

Pour compléter l'approche liée au sens et aux connaissances, différentes expériences sensibles et sensorielles (Ancel, 2015) sont aussi proposées : il s'agira alors de placer le visiteur au centre d'un dispositif ou d'une expérience, de l'amener à visiter en amateur, au sens d'Hennion (2004) et de sa sociologie des attachements, comme celui qui aime, découvre et prend goût. Ces médiations originales, liées aux thèmes présents dans l'œuvre de Rousseau, et faisant appel aux cinq sens, ont notamment été déployées lors du tricentenaire de sa naissance. Plus récemment, la programmation de 2017 décline des médiations multisensorielles destinées à faire découvrir les lieux, à procurer plaisir et détente, ou à faire mieux apprécier l'œuvre de Rousseau : lors des $13^{\text {es Rencontres }}$ philosophiques, des « siestes sonores » dans le jardin proposent deux parcours sonores téléchargeables, réalisés par des auteurs contemporains ${ }^{17}$ dans le cadre du projet européen "Lieux vivants ». En 2018, d'autres temps forts éditorialisent à nouveau la saison. Par la délectation, l'intention est de renouveler la curiosité et la connaissance de l'homme, de l'œuvre et du lieu. La « balade de Jean-Jacques », « balade gourmande et culturelle ${ }^{18}$, explore à elle seule la polysensorialité : découverte du piano-forte en 
concert, lecture de textes, dégustation de vins bio de Savoie ou du miel des Charmettes, découverte par les sens du jardin botanique. Ces médiations contemporaines constituent donc autant d'actions qui entretiennent, prolongent et réactualisent la patrimonialisation en acte, dans une plus ou moins grande proximité avec l'œuvre de Rousseau. Elles accompagnent le devenir-patrimoine de la Maison des Charmettes en rendant le lieu vivant, et en réinterprétant les thématiques chères au promeneur solitaire, qui avait pensé l'écriture d'une Morale sensitive.

\section{Conclusion}

En définitive, l'observation du processus de patrimonialisation dès son émergence, quoi qu'il en soit de sa temporalité, permet de mettre en relief la puissance patrimoniale que donne le politique comme le collectif et l'individu à un être ou un objet entendu comme exceptionnel pour son rôle dans la mémoire collective. Mais il éclaire aussi sa potentielle fragilité au cours du temps et des idéologies, d'où la nécessité de sans cesse le réactiver et de penser son avenir. L'exemple de la maison des Charmettes retrace l'histoire de la conservation d'une maison, et de sa valorisation, notamment par sa mise en mémoire et en exposition, jusqu'à sa transformation et sa labellisation en maison d'écrivain, creuset contemporain d'une mise en communication et d'une démarche de tourisme culturel et littéraire (Bonniot-Mirloup \& Blasquiet, 2016). Depuis la naissance du culte de Rousseau en son temps, en passant par les pèlerinages sur ses pas jusqu'aux commémorations, l'actualisation régulière du processus de patrimonialisation se traduit par les études sur l'œuvre ainsi que par la sauvegarde et la valorisation des lieux.

Patrimonialiser, c'est tenter d'échapper à l'usure du temps, faire circuler des objets dans le temps et ainsi transformer ces derniers et les représentations qui leur sont attachées. La patrimonialisation de cette maison s'élabore ainsi dans un processus symbolique de légitimation - par la figure de Rousseau - d'une part, et de coconstruction - par les visiteurs et les médiations - d'autre part. Dans l'évolution retracée, la figure de Jean-Jacques Rousseau apparaît comme un « être culturel » passé à la postérité, tandis que la maison des Charmettes représente un lieu privilégié de sa circulation, tous deux incarnant une forme de " trivialité » ou de " trivialisation », dans le sens d'Yves Jeanneret (2008), car si le philosophe est connu, comme de son vivant il en est beaucoup qui le visitent sans l'avoir lu.

40 La transformation de la maison en lieu de mémoire, davantage qu'en musée malgré l'appellation qui lui est parfois donnée, va-t-elle conduire davantage les visiteurs vers la lecture de l'œuvre? Tel est désormais l'enjeu pour que la vie de ce patrimoine perdure, car « ce qui justifie l'existence des maisons d'écrivain est [d'abord] dans des textes inaccessibles pendant le temps de la visite » (Menant, 2009 : 771). Le devenir de cette demeure et la transmission attachée à cette " filiation inversée » (Davallon, 2006) semblent ainsi reposer autant sur les conservateurs et médiateurs que sur les visiteurs et lecteurs.

\section{Bibliographie}

Ambrus (Gauthier) \& Grosrichard (Alain) (dir.). 2012. Vivant ou mort, il les inquiétera toujours. Amis et ennemis de Rousseau (XVIIIe-XXIe siècles). Catalogue de l'exposition de la Bibliothèque de Genève, de la Fondation Bodmer Cologny et du Musée Voltaire (20 avril - 16 sept. 2012). Gollion, Montricher : Infolio éditions et Éditions Noir sur Blanc.

Ancel (Pascale). 2015. « Histoire, mémoire, émotion. La place du sensible au musée », p. 31-42 in Les Mondes de la médiation culturelle / sous la direction de Cécile Camart, François Mairesse, Cécile Prévost-Thomas \& Pauline Vessely, vol. 2. Paris : L’Harmattan.

Barny (Roger). 1986. Rousseau dans la Révolution. Le personnage de Jean-Jacques et les débuts du culte révolutionnaire (1787-1791). Oxford : Voltaire Foundation.

Baudoin (Charles). 1962. «Pèlerinages ou de quelques étapes du Promeneur solitaire ». La Table ronde, 176 , p. 19-30. 
Berchtold (Jacques) \& Porret (Michel) (dir.). 1999. Rousseau visiteur, Rousseau visité. Les dernières années (1770-1778). Genève : Droz (Annales Jean-Jacques Rousseau).

Bonnet (Jean-Claude). 1998. Naissance du Panthéon. Essai sur le culte des grands hommes. Paris : Fayard.

Bonniot-Mirloup (Aurore) \& Blasquiet (Hélène). 2016. « De l'œuvre aux lieux : la maison d'écrivain pour passerelle (France) ». Territoire en mouvement, revue de géographie et d'aménagement [en ligne], 31 : http://tem.revues.org/3722 ; DOI : 10.4000/tem.3722 [consulté le 22 novembre 2019].

DOI : $10.4000 /$ tem.3722

Cussac (Hélène) \& Lambert (Emmanuelle). 2020a (à paraître). " Valeurs testimoniale et patrimoniale des livres d'or : les écrits de visiteurs de la maison-musée des Charmettes », in Les Témoins au musée. Mémoires du patrimoine mémoriel / sous la direction de Esteban CastanerMunoz, Michèle Gellereau \& Virginie Soulier. Perpignan : Presses universitaires de Perpignan.

Cussac (Hélène) \& Lambert (Emmanuelle). 2020b (à paraître). « Maisons d'écrivain et médiations sensibles : promenades imaginaires sur les pas de Rousseau, réminiscences émotionnelles sous les toits de Jean-Jacques », in Émotions littéraires, émotions patrimoniales. Actes du colloque de Saint-Denis organisé par Bérengère Voisin, Aurélie Mouton Rezzouk \& Sylvie Gonzales, 1-2 déc. 2016.

Davallon (Jean). 2006. Le Don du patrimoine : Une approche communicationnelle de la patrimonialisation. Paris : Hermes Science-Lavoisier.

Davallon (Jean). 2015. " Mémoire et patrimoine : pour une approche des régimes de patrimonialisation ", in Mémoire et nouveaux patrimoines [en ligne] / sous la direction de Cécile Tardy et Vera Dodebei, Marseille, OpenEdition Press : https://books.openedition.org/oep/444 [consulté le 20 novembre 2019].

DOI : $10.4000 / 1895.2$

Dekiss (Jean-Paul). 2009. " La maison d'un écrivain, utopie ou enjeu de société ». Revue d'histoire littéraire de la France, 109(4), p. 783-795.

DOI : 10.3917/rhlf.094.0783

Ferrand (Nathalie). 2012. "Les manuscrits de Rousseau », p. 30 in Jean-Jacques Rousseau et les arts / sous la direction de Guilhem Scherf. Paris : Éditions du Patrimoine.

Flon Émilie, 2014, "Introduction ». Culture \& Musées, 23, " Tourisme et médiations des patrimoines ", p. 13-20.

Forster (Elizabeth A.). 1921. Le Dernier Séjour de J.-J. Rousseau à Paris, 1770-1778. Paris : Champion.

Goulemot (Jean-Marie) \& Walter (Éric). 1984. « Les centenaires de Voltaire et de Rousseau. Les deux lampions des Lumières », p. 381-420, in Les Lieux de mémoire. I La République / sous la direction de Pierre Nora. Paris : Gallimard.

Guichard (Olivier). 2009. " Introduction ». Orages. Littérature et culture, 1760-1830, 8, "L’indispensable visite. Naissance du pèlerinage littéraire et artistique », p. 11-19.

Guillaume (James) (éditeur). 1891-1958. Procès-verbaux du Comité d'instruction publique de la Convention nationale. Paris : Imprimerie nationale (Collection de documents inédits sur l'histoire de France), 7 tomes.

Heinich (Nathalie). 2009. La Fabrique du patrimoine, "De la cathédrale à la petite cuillère ». Paris : Éd. de la Maison des sciences de l'homme.

Heinich (Nathalie). 2012. De la visibilité. Excellence et singularité en régime médiatique. Paris : Gallimard.

Heinich (Nathalie). 2013, "Esquisse d'une typologie des émotions patrimoniales », p. 195-210 in Émotions patrimoniales / sous la direction de Daniel Fabre. Paris : Éd. de la Maison des sciences de l'homme (Ethnologie de la France, 27).

Hennion (Antoine). 2004. "Une sociologie des attachements. D'une sociologie de la culture à une pragmatique de l'amateur ». Sociétés, 85, p. 9-23.

Jeanneret (Yves). 2008. Penser la trivialité. Vol. 1 La Vie triviale des êtres culturels. Paris : Hermes Science-Lavoisier (Communication, médiation et construits sociaux).

Kaehr (Roland). 2012. " La tenue de Rousseau était-elle arménienne ? ». Littera Edebiyat Yazılarl, 31, p. 77-90. En ligne : http://rousseaustudies.free.fr/articleKaehrrousseularmenien.pdf [consulté le 21 novembre 2019].

L’Aminot (Tanguy). 2009. " Un pèlerinage initiatique : le Voyage à l'Isle des Peupliers d'Arsène Thiébaut ». Orages. Littérature et culture, 1760-1830, 8, "L'indispensable visite. Naissance du pèlerinage littéraire et artistique », p. 51-66.

L’Aminot (Tanguy). 1992. Images de Jean-Jacques Rousseau de 1912 à 1978. Oxford : Voltaire Foundation.

Lilti (Antoine). 2014. Figures publiques. L’invention de la célébrité (1750-1850). Paris : Fayard. 
Menant (Sylvain). 2009. « Maison d'écrivain et histoire littéraire ». Revue d'histoire littéraire de

la France, 109(4), p. 771-781.

DOI : 10.3917/rhlf.094.0771

Mercier (Louis-Sébastien). 1791. De J. J. Rousseau, considéré comme l'un des premiers auteurs de la Révolution. Paris : Buisson, 2 vol.

Nora (Pierre). 2011. Présent, nation, mémoire. Paris : Gallimard.

Palluel-Guillard (André). 2012. " Rousseau et Chambéry, je t’aime, moi non plus », p. 27-45 in Autour de Jean-Jacques Rousseau. Chambéry : Altal Éditions (Société des Amis du vieux Chambéry, 20).

Poulot (Dominique) (dir.). 2001. "Défendre le patrimoine, cultiver l'émotion ». Culture \& Musées, 8.

Rautenberg (Michel) \& Tardy (Cécile). 2013. " Patrimoines culturels et naturels : analyse des patrimonialisations ». Culture \& Musées, hors-série, p. 115-138.

Raymond (Georges-Marie). 1811. Notice sur les Charmettes, vallon des environs de Chambéry ; à l'usage des voyageurs qui visitent la retraite de J. J. Rousseau. Genève, Paris : Paschoud.

Rousseau (Jean-Jacques). 1959. Les Confessions [1782 et 1789] in Euvres complètes. Vol. 1, sous la direction de Bernard Gagnebin et Marcel Raymond. Paris : Gallimard (La Pléiade).

Rousseau (Jean-Jacques). 1974. Correspondance complète de Jean-Jacques Rousseau. Édition de R. A. Leigh et al. Banbury: Voltaire Foundation, 52 vol.

Sacquin (Michèle). 2012. " Les manuscrits de Rousseau pendant la Révolution ». Revue de la $B n F, 42$, p. 56-69.

DOI : 10.3917/rbnf.042.0056

Sander (David) \& Varone (Carole). 2011. « L'émotion a sa place dans toutes les expositions ». La Lettre de l'OCIM [en ligne], 134, p. 22-28 : http://ocim.revues.org/840 ; DOI : 10.4000/ ocim.840 [consulté le 22 novembre 2019].

Saurier (Delphine). 2003. Médiations et co-construction du patrimoine littéraire de Marcel Proust, la Maison de tante Léonie et ses visiteurs. Thèse de doctorat en sciences de l'information et de la communication, Université d'Avignon et des Pays de Vaucluse.

Saurier (Delphine). 2007. « Proust dans ses meubles. Patrimonialisation de la Maison de tante Léonie ». Ethnologie française [en ligne], 37(3), p. 541-549 : https://www.cairn.info/revueethnologie-francaise-2007-3-page-541.htm [consulté le 22 novembre 2019].

Saurier (Delphine). 2013. La Fabrique des illustres. Proust, Curie, Joliot et lieux de mémoire. Paris : Éditions Non Standard (SIC : Recherches en sciences de l'information et de la communication).

Scherf (Guilhem) (dir.). 2012. Jean-Jacques Rousseau et les arts. Catalogue de l'exposition présentée au Panthéon du 29 juin au 30 septembre 2012. Paris : Éditions du Patrimoine.

Scipion (Sylvie-Marie). 2009. «Ouvrir les portes d'une maison d'écrivain... ». La Lettre de l'OCIM [en ligne], 125 : http://ocim.revues.org/252 ; DOI : 10.4000/ocim.252 [consulté le 22 novembre 2019].

DOI : $10.4000 /$ ocim.252

Schlobach (Jochen). 1984. "Un reportage sur Rousseau en 1763 ». Dix-Huitième Siècle, 16, p. 211-218.

DOI : $10.3406 /$ dhs.1984.1497

Staël (Germaine de). 1789. Lettres sur les ouvrages et le caractère de J. J. Rousseau. s. l. : s. n.

Staël (Germaine de). 1998. De la littérature, considérée dans ses rapports avec les institutions sociales [1800]. Édition d'Alex Blaeschke. Paris : Classiques Garnier.

Termolle (Michel). 2014. «Un jeune baron flamand à Môtiers ». Bulletin de l'Association JeanJacques Rousseau [Neuchâtel], 74, p. 5-18. En ligne : https://www.swedhs.org/actualite/neny.html [consulté le 21 novembre 2019].

Tornatore (Jean-Louis). 2010. « L'esprit de patrimoine ». Terrain, 55, p. 106-127.

DOI : $10.4000 /$ terrain.14084

Trousson (Raymond). 2004. Rousseau par ceux qui l'ont vu. Bruxelles : Éditions Le Cri.

Védrine (Mireille). 2012. "Quelques notes sur Georges-Marie Raymond et Jean-Jacques Rousseau », p. 59-67 in Autour de Jean-Jacques Rousseau. Chambéry : Altal Éditions (Société des Amis du vieux Chambéry, 20).

Watremez (Anne). 2008. "Vivre le patrimoine urbain au quotidien : pour une approche de la patrimonialité ». Culture \& Musées, 11, p. 11-36.

DOI : 10.3406/pumus.2008.1467

\section{Notes}


1 Si ce n'est pas aux Charmettes que Rousseau rédigea ses œuvres les plus connues, la maison en signe indéniablement la genèse ; aussi est-ce la raison pour laquelle elle revêt autant d’importance dans ce qui constitue le patrimoine littéraire du philosophe.

2 Elle est en effet présentée sous ce statut sur le site Internet des maisons d'écrivains et sur celui de terresdecrivains.com qui précise : "Les Charmettes sont une des plus anciennes maisons d'écrivains ouvertes au public » (consulté le 21 novembre 2019).

3 Voir la section «Portraits » du catalogue d'exposition Jean-Jacques Rousseau et les arts (Scherf, 2012 : 130-149).

4 Voir la section « Monuments » (ibid. : 158-169).

5 Auguste Castellant, " homme de lettres, journaliste et politicien radical consacra une part de sa vie au culte de Jean-Jacques. Il participa très activement à l'érection de toutes ces statues » (L’Aminot, $1992: 11$ ).

6 Voir Forster, 1921 ; Berchtold \& Porret, 1999 ; Trousson, 2004 ; Kaehr, 2012 ; Termolle, 2014.

7 " Les témoignages des lecteurs contemporains du récit furent assez nombreux », affirme Jochen Schlobach qui publie le texte à la suite de son article (1984: 214).

815 juillet 1770 (F. M. Grimm, Correspondance littéraire, philosophique et critique, t. 7, 17701772, Paris, Furne, 1829, p. 26-27, cité par Berchtold \& Porret, 1999 : 133).

9 Voir Ambrus \& Grosrichard, 2012 : 195-196.

10 Voir Mireille Védrine (2012 : 59-67) et André Palluel-Guillard (2012 : 27-45).

11 Précisons que les passages extraits des livres d'or sont ici inédits. Pour des analyses plus approfondies sur ce corpus, nous renvoyons à une publication à paraître : Cussac \& Lambert (2020a). Cette recherche a été effectuée à la mairie de Chambéry ; que soient remerciés ici Mireille Védrine et ses collègues pour leur soutien dans ce travail.

12 La dénomination « Les Charmettes, maison de Jean-Jacques Rousseau » semble la plus stable sur les supports de communication actuels.

13 Voir notamment : Poulot, 2001 ; Heinich, 2013.

14 Mireille Védrine, Projet culturel et scientifique des Charmettes, Chambéry, musées, 2009.

15 Notamment de reproductions de manuscrits, de couvertures d'éditions originales, de partitions de musique, de pages d'herbiers ou de lettres.

16 Les Charmettes, maison de Jean-Jacques Rousseau, programmation juin-septembre 2017 et mai-septembre 2018 ; notons que les activités et événements concernent la période estivale pour profiter du jardin, et que l'accès est majoritairement gratuit.

17 Gauz, "Jean-Jacques, œuf des Charmettes » ; Nathalie Gendrot, « Visitation », , parcours sonores à télécharger sur smartphone ou tablettes.

18 Remarquons que les médiations sont ici déléguées à des associations et des professionnels (organisme public ou producteur privé) spécialistes de leur domaine.

\section{Pour citer cet article}

Référence papier

Hélène Cussac et Emmanuelle Lambert, « Le devenir-musée de la maison des Charmettes de Jean-Jacques Rousseau : la patrimonialisation d'une demeure à l'épreuve du temps », Culture \& Musées, 34 | 2019, 55-80.

Référence électronique

Hélène Cussac et Emmanuelle Lambert, « Le devenir-musée de la maison des Charmettes de Jean-Jacques Rousseau : la patrimonialisation d'une demeure à l'épreuve du temps ", Culture \& Musées [En ligne], 34 | 2019, mis en ligne le 16 décembre 2019, consulté le 08 juin 2021. URL: http://journals.openedition.org/culturemusees/3632; DOI :

https://doi.org/10.4000/culturemusees.3632

\section{Auteurs}

\section{Hélène Cussac}

Université Toulouse III Paul Sabatier

Hélène Cussac, enseignante à l'Université Toulouse III Paul Sabatier, qualifiée maîtresse de conférences en 9e section, est membre du laboratoire Patrimoine-Littérature-Herméneutique $(\mathrm{PLH})$ de l'Université Toulouse Jean Jaurès. Spécialiste en histoire des idées et littérature du XVIIIe siècle, elle a récemment co-dirigé le $n^{\circ} 48$ de la revue Dix-Huitième Siècle : "Se retirer du monde », juin 2016. Elle a aussi réédité - en collaboration - l'ouvrage collectif Discours du corps au XVIIIe siècle, 2015. Elle participe actuellement à l'édition des œuvres complètes de Bernardin de Saint-Pierre, est en train d'éditer les actes d'un colloque qu'elle a organisé en mai 2016 sur 
une figure patrimoniale toulousaine (Loménie de Brienne), et vient d'organiser le

XXXIIle colloque international de la Sator sur le sonore (mai 2019).

Courriel : helene.cussac[at]univ-tlse2.fr

\section{Emmanuelle Lambert}

Université Toulouse III Paul Sabatier

Emmanuelle Lambert est maîtresse de conférences en sciences de l'information et de la

communication, et membre du Lerass (EA 827, équipe MICS) à I'Université Toulouse III Paul

Sabatier. Ses recherches portent sur la médiation culturelle, dans ses déclinaisons patrimoniales, muséales ou artistiques, et sur l'analyse des modalités de mise à contribution des publics. Outre de nombreuses publications autour de ces thématiques, elle a notamment co-dirigé le dossier «Art, mémoire et territoire », du $n^{\circ} 78$ de la revue Sciences de la société (2009).

Courriel : emmanuelle.lambert[at]iut-tlse $3 . f r$

\section{Droits d'auteur}

Culture \& Musées 